\title{
Activation of Akt/GSK3 $\beta$ and Akt/Bcl-2 signaling pathways in nickel-transformed BEAS-2B cells
}

\author{
JING-JU PAN ${ }^{1,2}$, QING-SHAN CHANG ${ }^{2}$, XIN WANG $^{2}$, YOUNG-OK SON ${ }^{2}$, \\ JIANKANG LIU ${ }^{2}$, ZHUO ZHANG ${ }^{2}$, YONG-YI BI ${ }^{3}$ and XIANGLIN SHI ${ }^{2}$ \\ ${ }^{1}$ Institute of Chronic Non-Communicable Disease, Hubei Provincial Center for Disease Control and Prevention, \\ Wuhan 430079, P.R. China; ${ }^{2}$ Graduate Center for Toxicology, College of Medicine, University of Kentucky, \\ Lexington, KY 40536-0305, USA; ${ }^{3}$ Department of Occupational and Environmental Health, \\ School of Public Health, Wuhan University, Wuhan 430071, P.R. China
}

Received May 11, 2011; Accepted June 17, 2011

DOI: $10.3892 /$ ijo.2011.1157

\begin{abstract}
The Akt signaling pathway has been implicated in a wide range of cellular functions involving cell survival and proliferation, angiogenesis, metabolism and cell migration. Accumulating evidence suggests that Akt perturbations play an important role in human malignancy. Here, we investigated Akt perturbation in nickel-transformed cells. Chronic treatment of human bronchial epithelial BEAS-2B cells with low doses of nickel chloride resulted in cell transformation demonstrated by anchorage-independent (AI) growth, increased cell growth and alterations of cell growth pattern. Western blot assays show that phosphorylation of Akt at Ser473, but not that of p38, JNK and ERK, was increased in nickel-transformed cells compared with controls. Inhibition of Akt or PI3K by pharmacological or biochemical interference suppressed nickel AI growth and cell growth of transformed cells. Activation of Akt led to inhibition of GSK $3 \beta$ by phosphorylation at Ser9 in nickel-transformed cells. In addition, two major anti-apoptotic proteins of the Bcl family, Bcl-2 and Bcl-XL, were increased in nickel-transformed cells. By employing the small interfering RNA technique (siRNA), our results showed that siRNA Akt attenuated the expression of Bcl-2 and Bcl-XL in nickel-transformed cells, indicating that induction of Bcl-2 and Bcl-XL was likely mediated through Akt. ROS generation was decreased in nickel-transformed cells compared with controls. Moreover, down-regulation of retinoblastoma protein $(\mathrm{Rb})$ was observed in nickel-transformed cells. Taken together, these findings demonstrate that activation of Akt, followed by GSK $3 \beta$ inhibition and $\mathrm{Bcl}-2, \mathrm{Bcl}-\mathrm{XL}$ up-regulation and decrease of ROS generation, along with a synergistic effect of $\mathrm{Rb}$ down-regulation may cause apoptosis resistance, contributing to the overall mechanism of nickel carcinogenesis.
\end{abstract}

Correspondence to: Dr Xianglin Shi, Graduate Center for Toxicology, College of Medicine, University of Kentucky, 234 Health Science Research Building, Lexington, KY 40536-0305, USA

E-mail: xshi5@email.uky.edu

Key words: nickel, cell transformation, signal pathway

\section{Introduction}

The serine/threonine kinase Akt is known as protein kinase B (PKB). Since its discovery as an oncogene of the mouse leukemia virus AKT8 and a homolog of protein kinase C, much progress has been made to explore its diverse cellular roles $(1,2)$. It is involved in regulating, such as cell size/growth, cell proliferation and survival, glucose metabolism, genome stability and neo-visualization (3). Enhancing the survival and growth of cells is one of critical roles for Akt. Therefore, Akt activation has been observed not only in many human cancers including breast, endometrium, prostate, lung, pancreatic, liver, ovarian and colorectal cancers $(4,5)$, but also in some malignant transformation (6-8).

Nickel is a ubiquitous environmental transition metal and is widely used in industrial and medical processes (9). Epidemiological and laboratory studies have implicated nickel compounds as carcinogens (10). As results, nickel compounds have been classified as established carcinogen to humans (Group 1) by the International Agency for Research on Cancer (IARC) in 1990 (10). Due to its weak mutagenesis, epigenetic changes have been implicated in nickel carcinogenesis (11-13). Disruption of cellular iron homeostasis by interfering with irondependent enzymes and generation of reactive oxygen species (ROS) also reportedly contribute to nickel carcinogenesis (14). In addition, induction of the hypoxia signaling pathway reportedly represents a key mechanism in nickel-initiated carcinogenesis $(13,15)$. Despite the progress, the molecular mechanisms underlying nickel-induced cell transformation, which is an essential step in tumor development, remains poorly understood.

Given the established role of Akt implicated in cancer and cell transformation, we investigated whether the Akt pathway is activated in nickel-transformed cells. In this study, we demonstrate that chronic exposure of low dose soluble nickel chloride $\left(\mathrm{NiCl}_{2}\right)$ induced transformation of human bronchial epithelial BEAS-2B cells. Akt, but not MAPK, was activated in nickel-transformed cells, followed by glycogen synthase kinase $3 \beta$ (GSK3 $\beta$ ) inhibition and up-regulation of the Bcl-2, Bcl-XL protein expression, and decrease of ROS generation. These processes may lead to apoptosis resistance, contributing to nickel-induced carcinogenesis. 


\section{Materials and methods}

Cell culture and other reagents. Immortalized, non-tumorigenic human bronchial epithelial cells, BEAS 2B were used in this study (15). Cells were cultured in Dulbecco's modified Eagle's medium (DMEM, Calbiochem) supplemented with $10 \%$ fetal bovine serum (FBS), $5 \%$ penicillin/streptomycin and $2 \mathrm{mM}$ L-glutamine (Invitrogen) at $37^{\circ} \mathrm{C}$ in a humidified atmosphere with $5 \% \mathrm{CO}_{2}$. Nickel cholroride $\left(\mathrm{NiCl}_{2}\right), \mathrm{LY} 294002$ and 5-(2-benzothiazolyl)-3-ethyl-2-[2-(methylphenylamino) ethenyl]-1-phenyl-1H-benzimidazolium iodide (B2311) were purchased from Sigma. Antibodies against Akt, GSK3 $\beta$, phospho-JNK, JNK, phospho-ERK, ERK, phospho-p53, p53 and $\beta$-actin were purchased from Santa Cruz Biotechnology, PTEN, Bcl-XL, phospho-Akt at Ser473, phospho-GSK3 $\beta$ at Ser9, phospho-mTOR at Ser2448, mTOR, phospho-Bad at Ser136, Bad, phospho-p38, p38, Rb from Cell Signaling, Bcl-2 from Dako, dephospho- $\beta$-catenin, $\beta$-catenin from Calbiochem and phospho-GSK3 $\beta$ at Tyr216 from Applied Biological Materials. Dihydroethidium (DHE) and 5-(and-6)-chloromethyl-2',7'-dichlorodihydrofluorescein diacetate acetyl ester $\left(\mathrm{CM}-\mathrm{H}_{2} \mathrm{DCFDA}\right)$ were from Invitrogen.

Establishment of nickel transformed cell line and soft agar colony formation assay. BEAS-2B cells were chronically and continuously cultured without or with $\mathrm{NiCl}_{2}$ at concentration of $25,50,75$ and $100 \mu \mathrm{M}$, respectively. Cell transformation assay was checked biweekly by performing soft agar colony formation assay after 2-month treatment with nickel. For soft agar colony formation assay, specifically, $2 \times 10^{4}$ cells were plated in $2 \mathrm{ml}$ of top agar medium (DMEM with 10\% FBS and $0.3 \%$ agar) and layered over $2 \mathrm{ml}$ of bottom agar medium (DMEM with 10\% FBS and 0.5\% agar) in 6-well plates in triple and were incubated at $37^{\circ} \mathrm{C}$ for $3-4$ weeks. Formed colonies $\geq 0.5 \mathrm{~mm}$ were counted. Chronically nickel-treated BEAS-2B cells had the ability of anchorage-independent (AI) growth after 6-month treatment.

Colony was picked up from $75 \mu \mathrm{M} \mathrm{NiCl}_{2}$ treated BEAS-2B cells with sterilized pipette and trypsinized in a 6 -well plate for $\sim 2 \mathrm{~min}$ and then trypsinization process was stopped by adding $2 \mathrm{ml}$ DMEM. Cells were cultured in 6-well plates and later transferred to flask when cell density reached $90 \%$ confluent. Cells derived from the colony were cultured and defined as nickel-transformed cells. These nickel-transformed cells were rechecked for colony formation to confirm their transformation properties and used for experiment later on. Paralleled cultured BEAS-2B cells without $\mathrm{NiCl}_{2}$ treatment were used as control cells.

Cell counting assay. Cell counting was carried out to assess cell proliferation between nickel-transformed BEAS-2B cells and control cells. Both control and nickel-transformed cells were seeded in each well of 6-well plates and grown for various timepoints as indicated. Then cells were washed by PBS, trypsinized and then cell counting was performed using Beckman Coulter.

Western blotting. NuPAGE Bis-Tris electrophoresis system from Invitrogen company was used to perform Western blot analysis. The total cellular samples were washed once with ice cold PBS and lysed in 1X RIPA buffer supplemented with
$50 \mathrm{mmol} / \mathrm{l}$ DTT (Fisher Biotech), then loaded with NuPAGE LDS sample buffer. The protein concentration was determined by Coomassie Protein Assay Reagent (Pierce). The total cellular protein extracts were separated by SDS-PAGE and transferred to nitrocellulose membrane. Membranes were blocked with 5\% fat-free dry milk in $1 \mathrm{X}$ TBS containing $0.05 \%$ Tween-20 and incubated with antibodies (all primary antibodies diluted at 1:1000, except 1:2000 for GSK3 $\beta$ and actin, 1:200 for phosphop38). Protein bands were detected by incubation with horseradish peroxidase-conjugated antibodies (1:4000, Kirkegaard and Perry Laboratories) and visualized with enhanced chemiluminescence reagent (Perkin-Elmer Life Sciences). Band density was performed using AlphaImager HP Imaging System from Alpha Innotech.

Cell transfection. To block Akt activation, the specific small interference RNA (siRNA) targeted Akt (which inhibits expression of Akt1 and 2, not Akt3) and control were purchased from Cell Signaling Co. Cell transfection was performed using Lipofectamine $^{\mathrm{TM}}$ RNAiMAX from Invitrogen Co. Transfection procedure was followed by the protocol provided by the transfection reagent manufacturer. Briefly, control siRNA and siRNA Akt were incubated with Lipofectamine ${ }^{\mathrm{TM}}$ RNAiMAX in OPTI-MEM I for $20 \mathrm{~min}$ at room temperature and then added to cells in maintenance media without antibiotics (final concentration of both control siRNA and Akt siRNA were $100 \mathrm{nM}$ each). Media were replaced with maintenance media with antibiotics $24 \mathrm{~h}$ later after transfection. Experiments were performed $\sim 48 \mathrm{~h}$ following transfection.

ROS measurement. ROS production was detected by using the fluorescent probe CM- $\mathrm{H}_{2}$ DCFDA (Molecular Probes) or DHE (Molecular Probes). CM- $\mathrm{H}_{2}$ DCFDA is oxidized to green fluorescent DCF (dichlorofluorescein) by $\mathrm{H}_{2} \mathrm{O}_{2}$ and DHE is oxidized to red fluorescent ethidium by $\mathrm{O}_{2}^{-{ }^{-}}(16)$. Cells were loaded with $5 \mu \mathrm{M} \mathrm{CM}-\mathrm{H}_{2} \mathrm{DCFDA}$ or $10 \mu \mathrm{M}$ DHE for $30 \mathrm{~min}$, respectively, at $37^{\circ} \mathrm{C}, 5 \% \mathrm{CO}_{2}$ in PBS. After washing twice, mean fluorescence intensity was determined as ROS generation by flow cytometry FACS Calibur (BD Bioscience, San Jose, CA).

Statistical analysis. For statistical analysis, values were presented as mean \pm SE. Statistical differences between controls and treated groups were determined by One Way ANOVA. Differences were considered statistically significant for $\mathrm{P}<0.05$.

\section{Results}

Chronic exposure of low dose nickel induces transformation of BEAS-2B cells. We first tested if low dose of nickel chloride could induce transformation of BEAS-2B cells in vitro. BEAS-2B cells were continuously exposed to $0,25,50,75$ and $100 \mu \mathrm{M}$ nickel chloride for 6 months. During the period, cell transformation was checked biweekly by performing soft agar colony formation assay. Our study showed that BEAS-2B cells treated with different concentration of nickel gained the ability of anchorage-independent (AI) growth after 6-month treatment, while control cells did not acquire the ability to grow in soft agar or died shortly after plating (Fig. 1A). Fig. 1B demonstrates that different concentrations of nickel all conferred AI growth capacity of BEAS-2B cells. 
A
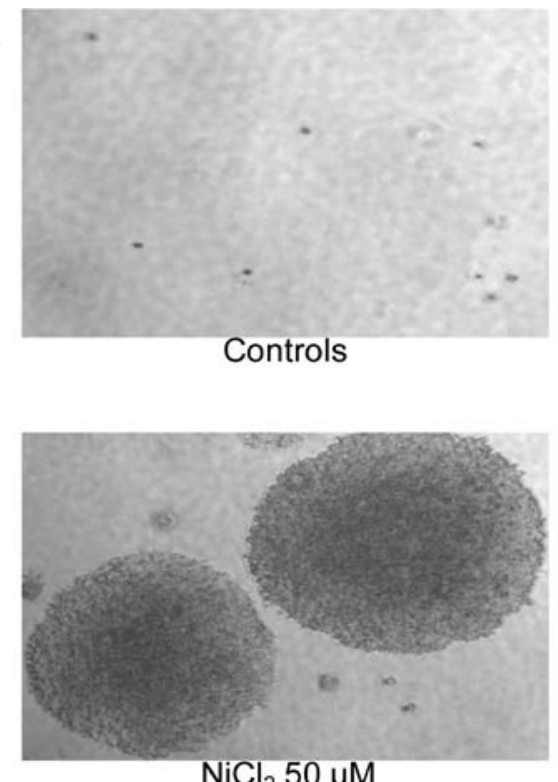

$\mathrm{NiCl}_{2} 50 \mu \mathrm{M}$

B

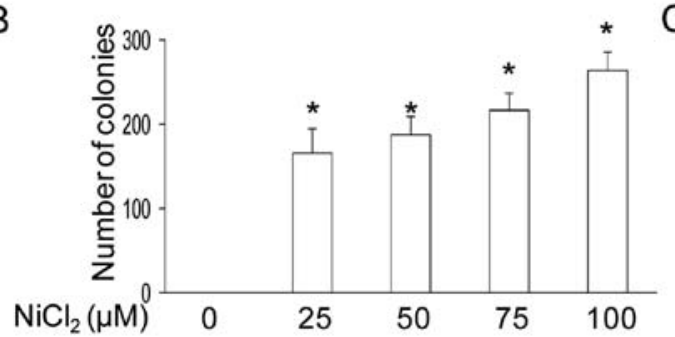

D

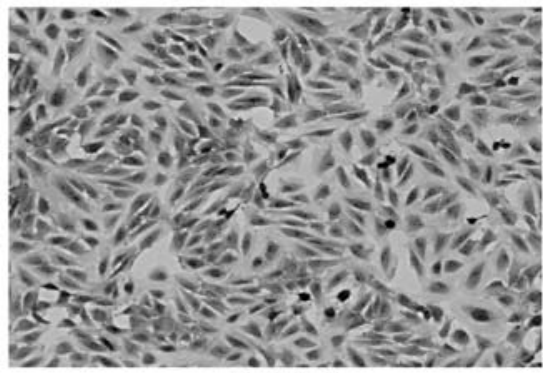

Control

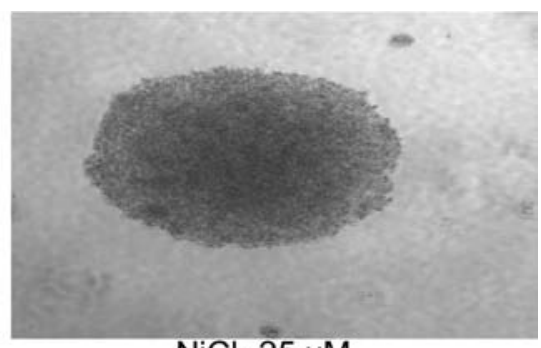

$\mathrm{NiCl}_{2} 25 \mu \mathrm{M}$

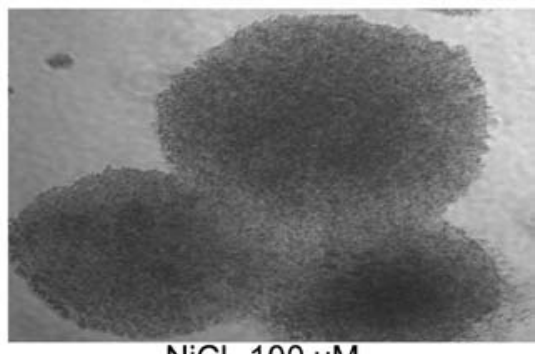

$\mathrm{NiCl}_{2} 100 \mu \mathrm{M}$
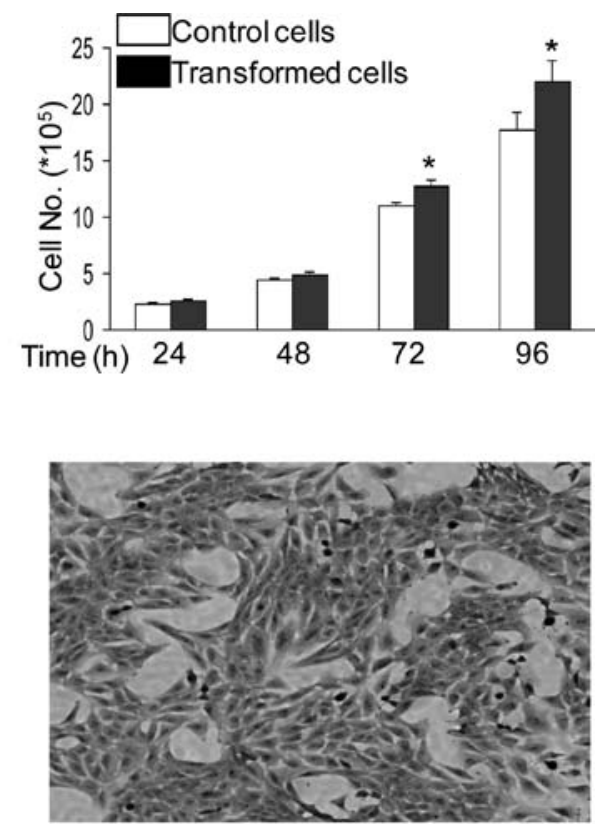

Transformed

Figure 1. Low dose of $\mathrm{NiCl}_{2}$ induces transformation of BEAS-2B cells. (A) Different low dose of $\mathrm{NiCl}_{2}$ all induced colony formation in soft agar. The data shown here are typical examples of colonies formed in soft agar from three independent experiments. Photomicrographs of colonies were taken 3 weeks after plating (x10). Cell treatment is described in Materials and methods in detail. (B) Quantitative analysis of colony formation in soft agar. Bars represent the averages $( \pm \mathrm{SD})$ of three independent experiments. The number of colonies in soft-agar was counted 3 weeks after plating. ${ }^{*} \mathrm{P}<0.05$, significance between control cells and nickel-transformed cells. (C) Comparison of proliferation growth rate between control cells and nickel-transformed cells. Cells $\left(1.5 \times 10^{5}\right)$ were plated in 6-well plates and cultured for indicated time-point, then cells were washed by PBS, typsinized and counted using Beckman Coulter. * $<0.05$ significance between control cells and nickel-transformed cells. (D) Alterations in cell growth pattern between control cells and nickel-transformed cells. Cells ( $\left.2 \times 10^{5}\right)$ were plated in 60-mm dishes and cultured for $48 \mathrm{~h}$, and then pictures were taken using phase contrast microscope (x4) and representative images of three independent experiments are shown.

As described in Materials and methods in detail, cells isolated from colony grown in soft agarose were defined as nickel-transformed cells and utilized for later experiments. These nickel-transformed cells were re-examined for AI growth to confirm their ability of growing in soft agar. As expected, these nickel-transformed cells formed colonies in soft agar (data not shown). One of the key characteristics of transformed cells is their increased ability to survive when comparing to their normal counterparts. Fig. 1C shows that nickel-transformed cells also proliferated faster than control cells. Morphological changes have been reported in some nickel-treated cells $(17,18)$. Cells undergo from epithelial-like to less epithelial or fibroblast-like, but in nickel-transformed BEAS-2B cells, morphological alterations were not obvious compared with control cells (Fig. 1D). However, the pattern of cell growth changed for those nickel-transformed 


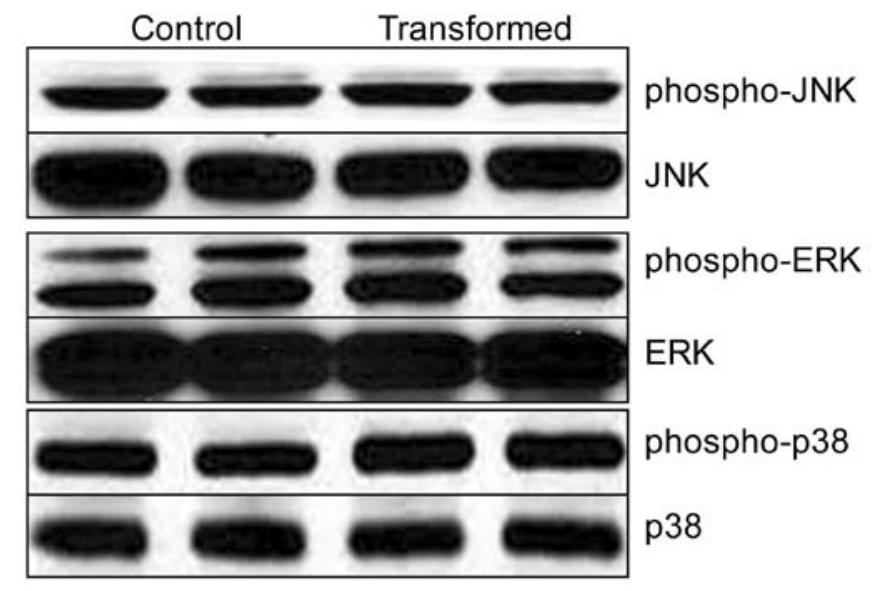

Figure 2. Analysis of the MAPK activation in nickel-transformed cells. Total cell lysates from both control and nickel-transformed cells were subjected to immunoblot analysis with antibody specific for the phosphorylated form of p38, JNK and ERK, respectively. The same blots were also stripped first and reprobed with antibody to p38, JNK and ERK to confirm that equal amounts of these proteins were loaded. All immunoblot analyses performed in the current study are representatives of at least three independent experiments.

BEAS-2B cells. They grew in clustered and aggregated forms (Fig. 1D).

Akt pathway, not the MAPK pathway, is activated in nickeltransformed BEAS-2B cells. Among the complicated signaling network, Akt and MAPK pathways are two well-defined signaling pathways involved in cell transformation. We first examined potential involvement of the MAPK pathway. Phosphorylation of endogenous p38, ERK and JNK, which represents the activated states of the kinases, was analyzed by immunoblotting. No significant difference was found between nickel-transformed and control cells (Fig. 2), suggesting that the MARK pathway was not activated in nickel-transformed BEAS-2B cells.

Using the same strategy, we analyzed the activation state of endogenous Akt protein in both nickel-transformed and control cells by immunoblotting. There was obvious difference in the phosphorylation of Akt at Ser473, which represents activated Akt (19), between transformed and control cells (Fig. 3A). B2311, an Akt inhibitor that inhibits Akt phosphorylation/activation, has also been used (20). Our results showed that treatment of nickel-transformed cells with B2311 has reduced cell growth and decreased colony formation in soft agar (Fig. 3D and E). Thus, Akt was constitutively activated in nickel-transformed BEAS-2B cells.

Akt activation regulated by $P I 3 K$, not PTEN. Akt is a major effector of phosphoinositide 3-kinase [PI(3)K] (21). We tested whether Akt phosphorylation in nickel-transformed cells is mediated through PI3K. As shown in Fig. 3B, LY294002, a wellestablished PI3K inhibitor, partially suppressed the nickelinduced Akt phosphorylation, implying that nickel-induced constitutive activation of Akt is, at least in part, mediated via PI3K. We also examined the effect of PI3K inhibitor, LY294002, on cell proliferation and colony formation of nickel-transformed cells. Our results demonstrated that inhibition of PI3K dramati- cally attenuated the cell proliferation and colony formation of nickel-transformed cells (Fig. 3D and E).

PTEN (phosphatase with tensin homology) is a lipid phosphatase and a tumor suppressor that negatively regulates the Akt signaling pathway (22). Since Akt activation is partially mediated by PI3K, we tested if PTEN is involved in the Akt pathway in nickel-transformed cells. Our results showed similar expression of PTEN protein among nickel transformed and control cells (Fig. 3C).

Downstream targets of Akt are involved in nickel-transformed cells. Akt plays diverse roles through its substrates (21). The protein kinase mTOR (mammalian target of rapamycin) is phosphorylated at Ser2448 via the PI3 kinase/Akt signaling pathway (23). Dysregulation of Akt/mTOR pathway has been documented in a variety of transformed cells and human tumors (24). We then investigated the activation level of mTOR by performing Western blot analysis. However, both phosphorylation of mTOR at Ser2448 and mTOR were not significantly different between nickel-transformed and control cells (Fig. 4B).

However, phosphorylation of GSK3 $\beta$ at Ser9, another substrate and effector of Akt, was elevated in nickel-transformed BEAS-2B cells (Fig. 4A). GSK3 $\beta$ is a multifunctional serine/threonine kinase found in all eukaryotes. It is now known that GSK3 $\beta$ functions not only in diverse cellular processes including proliferation, differentiation, motility and survival, but also in neoplastic transformation and tumor development. Full activity of GSK3 $\beta$ generally requires phosphorylation at tyrosine (Tyr216), and conversely, phosphorylation at serine (Ser9) inhibits GSK3 $\beta$ activity (25). However, in nickel-transformed cells, phosphorylation of GSK3 $\beta$ at tyrosine (Tyr216) remained unchanged compared with parental cells (Fig. 4A). Therefore, our study demonstrated the inhibition of GSK3 $\beta$ activity in nickel-transformed cells.

Amounting evidence suggests that the Wnt/ $\beta$-catenin signaling pathway is often involved in oncogenesis and cancer development. GSK-3 $\beta$ is an essential component and regulator of the pathway, and activation of this kinase results in inhibition of the Wnt signaling pathway. Because GSK $3 \beta$ was inhibited in nickel-transformed cells, we examined if $\beta$-catenin is also activated in nickel-transformed cells. Dephosphorylated $\beta$-catenin and total $\beta$-catenin were expressed in both nickel-transformed and the control cells at similar levels (Fig. 4C).

Up-regulation of Bcl-2 and Bcl-XL in nickel-transformed cells mediated by Akt pathway. The Bcl-2 family of proteins plays an instrumental role in the regulation of apoptosis. This family includes both proapoptotic proteins such as Bad and Bax as well as anti-apoptotic proteins such as Bcl-2 and Bcl-XL. Bad is one of well known substrates of Akt. Phosphorylation of Bad by Akt at Ser136 can suppress apoptosis and promote survival (26). However the state of both phosphorylated Bad at Ser136 and total Bad showed no difference between nickel-transformed and control cells (data not shown). Since Bcl-2 and Bcl-XL are overexpressed in a variety of cancers (27), we investigated whether Bcl-2 and Bcl-XL were involved in nickel-induced cell transformation. Our study showed that protein expression of both Bcl-2 and Bcl-XL was up-regulated in nickel-transformed 
A
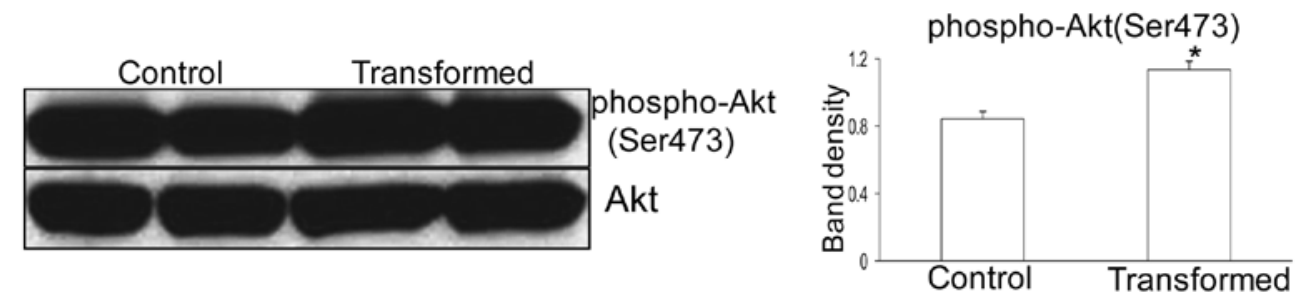

B

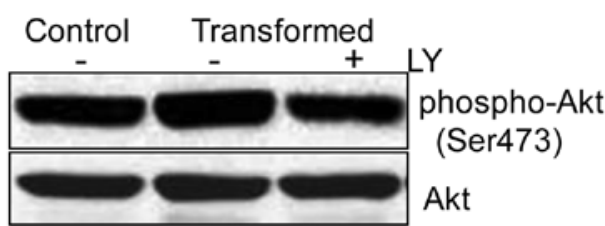

C
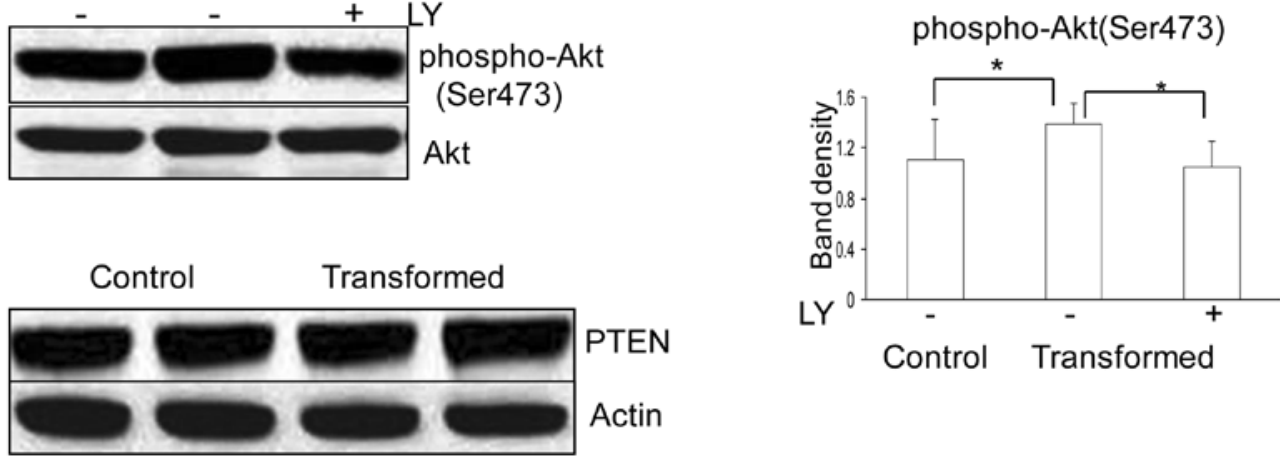

D

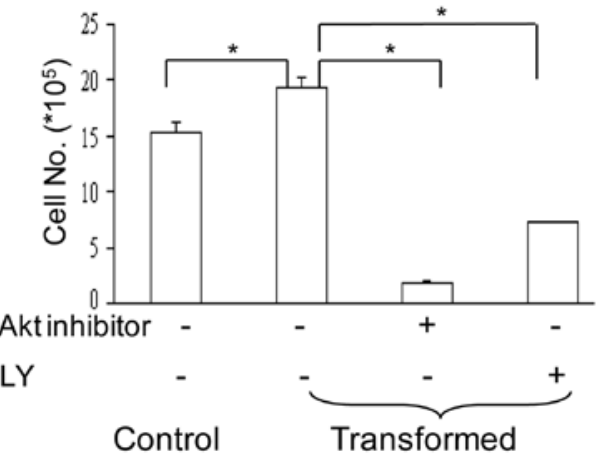

E

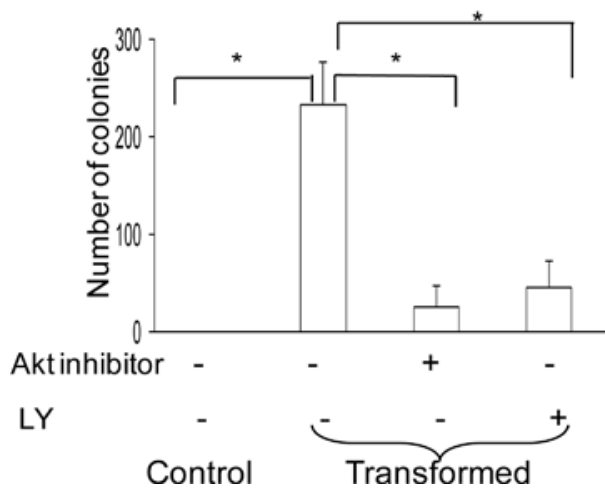

Figure 3. Activation of the PI3K/Akt in nickel-transformed cells. (A) Activation of Akt in nickel-transformed cells. Total cell lysates from both control and nickel-transformed cells were subjected to immunoblot analysis with antibody specific for the phosphorylated form of Akt. The same blot was also stripped first and reprobed with antibody to Akt to confirm that equal amounts of proteins were loaded. Quantitative analysis of band density is shown in the right panel normalized to Akt. Each bar represents the mean $\pm \mathrm{SE}$ of three independent experiments. ${ }^{*} \mathrm{P}<0.05$, significance between control cells and nickel-transformed cells. (B) Effects of PI3K inhibitor on Akt activation. Nickel-transformed cells were treated with or without LY294002 at $10 \mu \mathrm{M}$ for $48 \mathrm{~h}$. Then, total cell lysates from these cells as well as from control cells were subjected to immunoblot analysis with antibody specific for the phosphorylated form of Akt. The same blot was also stripped first and then reprobed with antibody to Akt to confirm that equal amounts of proteins were loaded. Quantitative analysis of band density is shown in the right panel. " $\mathrm{P}<0.05$, significance between control cells and nickel-transformed cells or between the indicated two groups. (C) Protein expression of PTEN in nickel-transformed cells. Total cell lysates from both control and nickel-transformed cells were subjected to immunoblot analysis with antibody specific for PTEN. The same blot was also stripped first and reprobed with antibody to actin to confirm that equal amounts of proteins were loaded. (D) Effects of PI3K inhibitor and Akt inhibitor on proliferation of nickel-transformed cells. Cells $\left(1.5 \times 10^{5}\right)$ were plated in 6-well plates. Nickel-transformed cells were cultured for $96 \mathrm{~h}$ with or without existence of PI3K inhibitor, LY294002 at $10 \mu \mathrm{M}$ and Akt inhibitor, B2311 at $1 \mu \mathrm{m}$. Then cells were washed by PBS and typsinized, followed by cell counting using Beckman Coulter. ${ }^{*} \mathrm{P}<0.05$, significance between control cells and nickel-transformed cells or between the indicated two groups. (E) Suppression of nickel-induced soft agar colony formation by PI3K inhibitor and Akt inhibitor. Nickel-transformed cells were treated with PI3K inhibitor, LY294002 at $10 \mu \mathrm{M}$ and Akt inhibitor, B2311 at $1 \mu \mathrm{M}$ for $72 \mathrm{~h}$, and then soft agar colony formation assay was performed according to methods described in Materials and methods. ${ }^{\mathrm{P}}<0.05$, significance between control cells and nickel-transformed cells or between the indicated two groups.

cells compared with the controls (Fig. 5). Furthermore, by using small interference RNA (siRNA) specific for Akt, we investigated whether up-regulation of Bcl-2 and Bcl-XL was mediated by Akt signaling pathway. Our results demonstrated that expression of both Bcl-2 and Bcl-XL proteins was apparently attenuated by siRNA Akt, implying that nickel-induced $\mathrm{Bcl}-2$ and Bcl-XL overexpression was regulated via the Akt pathway (Fig. 6).

Decrease of ROS generation in nickel-transformed cells. ROS, key molecules in signaling cascades, have been investigated in great detail on their involvement in cancer and cell transforma- tion over the last 10-15 years. A significant ROS generation has been found in many types of cancer cells (28). A review from the Cotter's group at the University College Cork in Ireland has emphasized the effect of ROS on PI3K/Akt signaling pathway in tumors (29). Consequently, we investigated ROS generation in nickel-transformed cells. However, we found that ROS generation, instead of increase, decreased in nickel-transformed cells compared with their counterparts (Fig. 7).

Down-regulated $R b$ protein, not p53 in nickel-transformed cells. Similar to carcinogenesis, cell transformation is also a multistep process. Activation of oncogenes and inactivation 


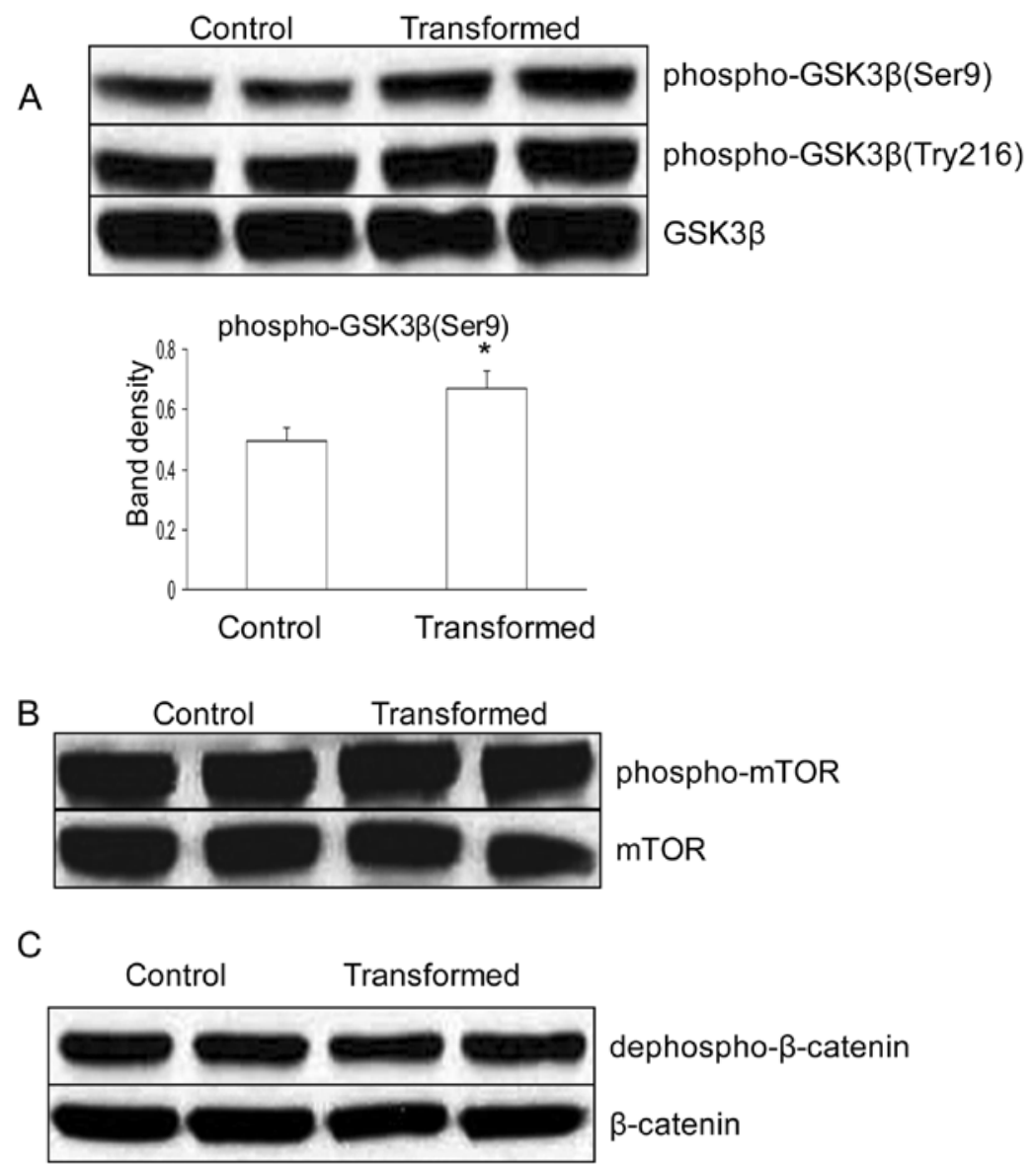

Figure 4. Downstream targets of Akt in nickel-transformed cells. (A, B) GSK3 $\beta$, not mTOR, is involved in the Akt pathway in nickel-induced transformation. Total cell lysates from both control and nickel-transformed cells were subjected to immunoblot analysis with antibody specific for the phosphorylated form of mTOR and GSK3 $\beta$ at Ser9 and Tyr216, respectively. The same blots were also stripped first and reprobed with antibody to mTOR and GSK3 $\beta$ to confirm that equal amounts of these proteins were loaded. (C) $\beta$-catenin is not involved in nickel-induced cell transformation. Total cell lysates from both control and nickel-transformed cells were subjected to immunoblot analysis with antibody specific for the dephosphorylated form of $\beta$-catenin. The same blots were later stripped first and reprobed with antibody to $\beta$-catenin to confirm that equal amounts of these proteins were loaded.

of tumor suppressor genes are involved in the process of cell transformation (30). Rb and the p53, two tumor suppressors, are the most prominent among the regulators disrupted in cancer cells. Western blot analysis showed down-regulation of $\mathrm{Rb}$ protein in nickel-transformed BEAS-2B cells compared with their counterparts (Fig. 8), suggesting a synergistic role for $\mathrm{Rb}$, along with activation of Akt pathway in nickel-transformed cells. Whereas, alterations of both phosphorylated p53 and p53 expression were not detected in nickel-transformed cells compared with their parental cells (Fig. 8).

\section{Discussion}

Both soluble and insoluble nickel compounds have been reported to be able to induce cell transformation in mouse and human cells including mouse embryo fibroblasts, HOS cells, and human bronchial epithelial (16HBE) cells, with relatively high concentrations of $\mathrm{NiCl}_{2}$ (1 or $\left.2 \mathrm{mM}\right)(31,32)$. Here we show that various concentrations of low dose $\mathrm{NiCl}_{2}$ ranging from 25 to $100 \mu \mathrm{M}$, were able to induce transformation of BEAS-2B cells as confirmed by AI growth, one of characteristics for transformed cells, which is a traditionally accepted criterion to confirm cell transformation. Moreover, nickel-transformed cells showed
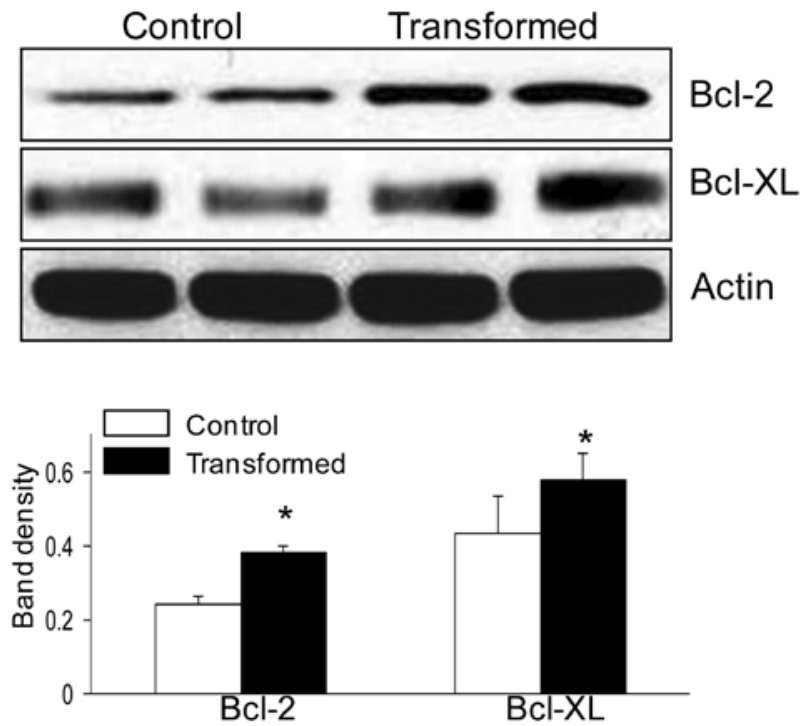

Figure 5. Up-regulation of Bcl-2 and Bcl-XL in nickel-transformed cells Total cell lysates from both control and nickel-transformed cells were subjected to immunoblot analysis with antibody specific for Bcl-2 and Bcl-XL. The same blots were also stripped first and reprobed with antibody to actin to confirm that equal amounts of these proteins were loaded. 
A
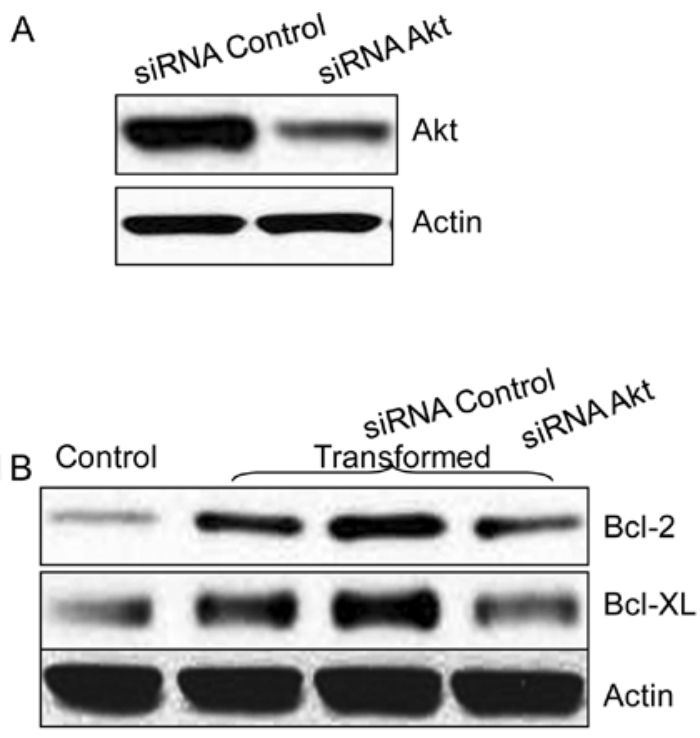
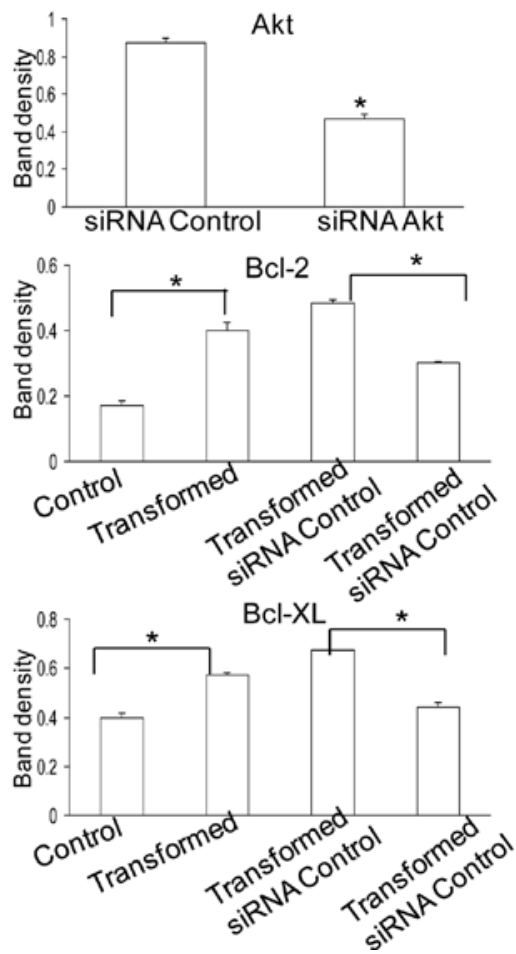

Figure 6. Up-regulation of Bcl-2 and Bcl-XL in nickel-transformed cells is mediated through Akt pathway. (A) siRNA Akt decreases Akt expression. Nickeltransformed cells were transfected with Akt siRNA (100 nM) and control siRNA (100 nM) for $48 \mathrm{~h}$. The protein expression was measured by immunoblot analysis. The results demonstrated are representative of three separate experiments. (B) siRNA Akt attenuated protein expression of Bcl-2 and Bcl-XL in nickel-transformed cells. Nickel-transformed cells were transfected with $100 \mathrm{nM}$ Akt siRNA and control siRNA for $48 \mathrm{~h}$. Total cell lysates from these cells were subjected to immunoblot analysis with antibody specific for Bcl-2 and Bcl-XL. The same blots were also stripped first and reprobed with antibody to actin to confirm that equal amounts of these proteins were loaded. Data are representative of three separate experiments.

A

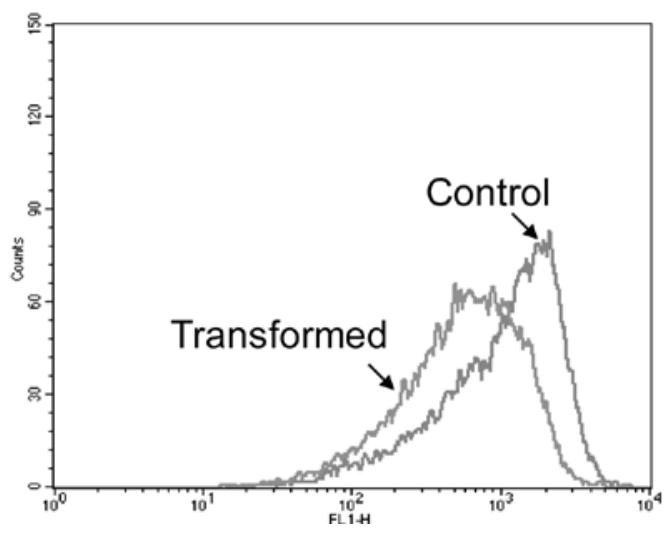

B

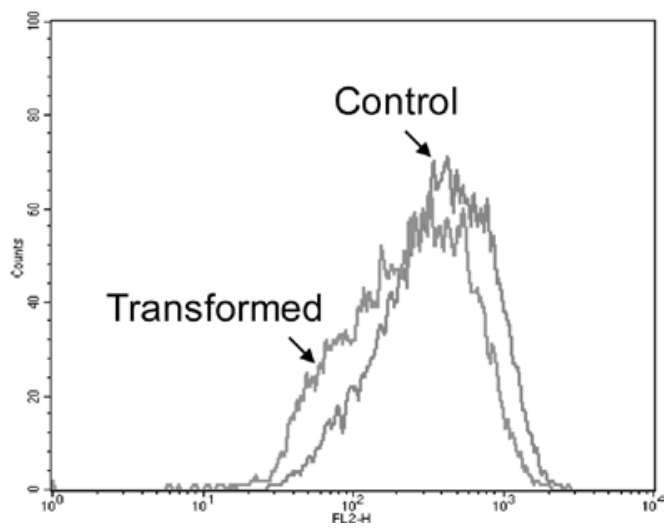

Figure 7. Generation of ROS in both nickel-transformed cells and controls. Both nickel-transformed and control cells $\left(4 \times 10^{5}\right)$ were seeded in a $60-$ mm dish. When cell density was about $90 \%$ confluent, the cells were labeled with CM-H2DCFDA (A) and DHE (B), respectively, followed by flow cytometry as described in Materials and methods. The rightward shift of the overlay reflects ROS generation. Illustrated overlays are representatives of at least three separate experiments.

accelerated growth and altered pattern of cell growth. Similar to our observations, Lin and Costa have observed this phenomenon in immortalized human osteoblastic (HOS) cells transformed by NiS (17). Nickel-transformed BEAS-2B cells grew in clustered pattern which might be a growth advantage by providing more space for nickel-transformed cells to grow to high densities.
The mechanism by which nickel induces cell transformation remains unclear. Previous studies by others have revealed the importance of gene expression and epigenetic alterations in nickel-induced cell transformation (14,31-35). However, little weight has been given to the signaling pathway alterations in nickel-induced cell transformation. 

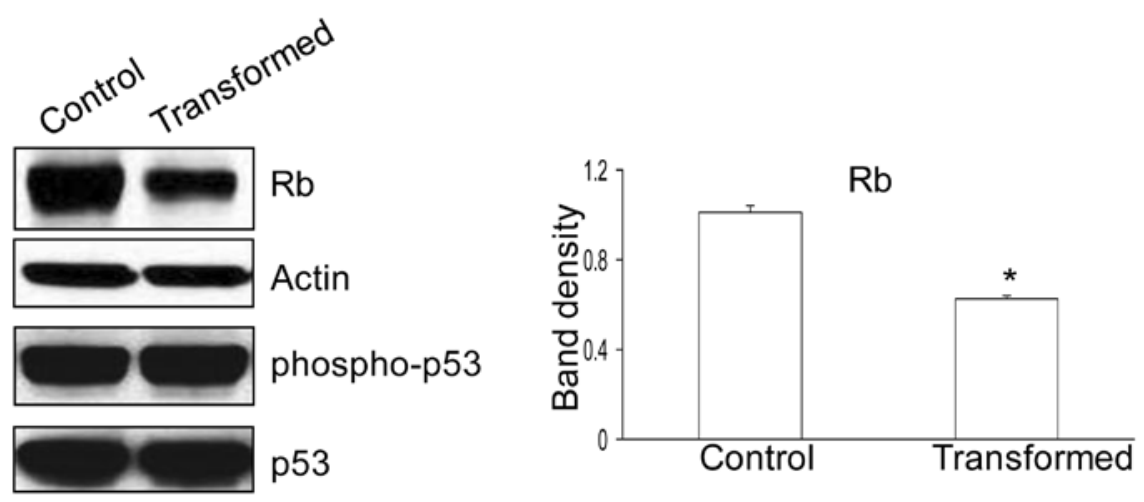

Figure 8. Down-regulation of Rb, not p53, is involved in nickel-induced cell transformation. Total cell lysates from both control and nickel-transformed cells were subjected to immunoblot analysis with antibody specific for $\mathrm{Rb}$ and phospho-p53, respectively. The same blots were also stripped first and reprobed with antibody to actin and p53, repectively to confirm that equal amounts of these proteins were loaded. Bars shown in the right panel are quantitative analysis of band density of $\mathrm{Rb}$ protein normalized to actin.

Studies reveal that activation of Akt pathway is required for cells undergoing transformation elicited by obl oncogene, FLJ10540 (6,36). We found that the PI3K/Akt pathway was constitutively activated in nickel-transformed BEAS-2B cells. By utilizing pharmacological inhibition method, we confirmed that LY294002 and B2311, inhibitor of PI3K and Akt, respectively, significantly suppressed proliferation of nickel-transformed cells, demonstrating the involvement of $\mathrm{PI} 3 \mathrm{~K} / \mathrm{Akt}$ pathway in nickel-induced transformation. The lipid phosphatase activity of PTEN is associated with down-regulation of multiple downstream components of the PI3K pathway, including Akt and its downstream targets. Level of PTEN is often correlated to activation of Akt. However, our results show that PTEN protein expression, which reflects PTEN activation, remains similar between nickel-transformed and control cells, probably suggesting that PTEN is not involved in activation of PI3K/Akt pathway.

Many substrates of Akt have been identified, mTOR is one of them. Activation of mTOR regulated by Akt is highly implicated in cancer. It has reported that Akt/mTOR pathway is also involved in cell transformation induced by EGF in JB6 cells (37). But in nickel-transformed BEAS-2B cells, elevated activation of mTOR was not observed. Akt enhances the survival of cells by blocking the function of pro-apoptotic proteins and processes and thereby contributes to apoptosis resistance. GSK3 $\beta$ was the first substrate identified to be phosphorylated and inhibited by Akt in 1995 (38). Akt can phosphorylate constitutively active GSK3 $\beta$ within the $\mathrm{N}$-terminal on serine 9 and block its apoptotic signaling. Phosphorylation on tyrosine 216 residue (Tyr216) is necessary for the functional activity of GSK3 $\beta$ (25). Research has revealed the implication of Akt/GSK3 pathway in growth regulation of myeloma cells, pancreatic cancer cells, vascular smooth muscle cells and IGF-1-induced skeletal myotube hypertrophy (37-40). Our current study shows that phosphorylation of GSK3 $\beta$ at Ser9 but not Tyr216 was apparently increased in nickel-transformed cells.

GSK3 $\beta$ can regulate diverse substrates and signaling pathways. One of the most important impacts of GSK $3 \beta$ on neoplastic transformation of tumor development is likely mediated by its influence on $\mathrm{Wnt} / \beta$-catenin signaling. Phosphorylation of $\beta$-catenin by active GSK3 $\beta$ targets $\beta$-catenin for ubiquitin- mediated proteasomal degradation (39). Activation of Wnt signaling inhibits GSK3 $\beta$ and stabilizes cytoplasmic $\beta$-catenin, qualifying $\beta$-catenin as a proto-oncogene (40). Since GSK3 $\beta$ was inhibited in nickel-transformed cells, we speculate a possible crosstalk between the Akt and Wnt pathways in nickelinduced cell transformation converging on GSK3 $\beta$. However, we did not observe obvious difference in the amounts of both dephosphorylated $\beta$-catenin and $\beta$-catenin expression between transformed and control cells.

Many members of the human Bcl-2 family proteins have been identified and alterations in their expression and function contribute to the pathogenesis and progression of human cancers (27). For instance, the Bcl-2 gene is elevated in perhaps half of all human cancers (27). Our study demonstrates that expression of Bcl-2 and Bcl-XL, two anti-apoptotic proteins of the Bcl family was elevated in nickel-transformed cells. Furthermore, to examine the role of Akt on expression of both $\mathrm{Bcl}-2$ and Bcl-XL, siRNA specific for Akt was carried out. Results show that expression of both Bcl-2 and Bcl-XL was attenuated by siRNA Akt, indicating that expression of $\mathrm{Bcl}-2$ and $\mathrm{Bcl}-\mathrm{XL}$ was mediated through the Akt pathway. It has been reported that Akt-dependent Bcl-XL overexpression was involved in acidic preconditioning protection of endothelial cells from apoptosis (41), while Akt could also up-regulate Bcl-2 expression and mediated cell survival signal in PC cells (42). Others have observed a synergistic effect between PI3K/Akt and Bcl-XL in controlling cell death (43).

Transformation is a complicated process and many factors are involved including oncogenes and suppression genes. Several lines of evidence support the hypothesis that tumor suppressor genes or oncogenes play synergistically a role in cell transformation induced by various inducers. For example, Myc has reported synergized with Ras and PI3-kinase to induce cell transformation (44). Here we found protein expression of $\mathrm{Rb}$ was down-regulated in nickel-transformed cells. $\mathrm{Rb}$ was the first identified tumor suppressor gene. It is mutated or expressed at very low levels in several tumor types, including retinoblastoma and osteosarcoma, as well as small cell lung, colon, prostate, bladder and breast carcinoma (45). Deregulation of $\mathrm{Rb}$ has also contributed to cell transformation (46). Similar to our observation, another study also found the 
hypophosphorylated $\mathrm{Rb}$ protein in NiS-transformed HOS cells (47). Even though abnormality of p53 is frequently observed in most human cancer, both phosphorylated p53 and total p53 remained unchanged between nickel-transformed cells and their counterparts.

In summary, our study shows that nickel is able to induce transformation of BEAS-2B cells. In nickel-transformed cells, Akt and not MAPK was activated, followed by GSK3 $\beta$ inhibition and up-regulation of Bcl-2, Bcl-XL, and decrease of ROS generation. These processes will lead to the apoptosis resistance, contributing to the overall mechanism of nickel-induced carcinogenesis.

\section{Acknowledgements}

X.S. is grateful for research support from NIH Grants RO1 ES015518-01A1.

\section{References}

1. Bellacosa A, Testa JR, Staal SP and Tsichlis PN: A retroviral oncogene, akt, encoding a serine-threonine kinase containing an SH2-like region. Science 254: 274-277, 1991.

2. Franke TF: PI3K/Akt: getting it right matters. Oncogene 27: 6473-6488, 2008.

3. Bellacosa A, Kumar CC, Di Cristofano A and Testa JR: Activation of AKT kinases in cancer: implications for therapeutic targeting. Adv Cancer Res 94: 29-86, 2005.

4. Altomare DA and Testa JR: Perturbations of the AKT signaling pathway in human cancer. Oncogene 24: 7455-7464, 2005.

5. Hennessy BT, Smith DL, Ram PT, Lu Y and Mills GB: Exploiting the PI3K/AKT pathway for cancer drug discovery. Nat Rev Drug Discov 4: 988-1004, 2005.

6. Morley S, Wagner J, Kauppinen K, Sherman M and Manor D: Requirement for Akt-mediated survival in cell transformation by the dbl oncogene. Cell Signal 19: 211-218, 2007.

7. Bedogni B, Welford SM, Cassarino DS, Nickoloff BJ, Giaccia AJ and Powell MB: The hypoxic microenvironment of the skin contributes to Akt-mediated melanocyte transformation. Cancer Cell 8: 443-454, 2005.

8. Sun M, Wang G, Paciga JE, et al: AKT1/PKBalpha kinase is frequently elevated in human cancers and its constitutive activation is required for oncogenic transformation in NIH3T3 cells. Am J Pathol 159: 431-437, 2001.

9. Oller AR, Costa M and Oberdorster G: Carcinogenicity assessment of selected nickel compounds. Toxicol Appl Pharmacol 143: 152-166, 1997.

10. International Agency for Research on Cancer (IARC): Nickel and nickel compounds. Summ Eval 49: 257, 1990.

11. Ke Q, Davidson T, Chen H, Kluz T and Costa M: Alterations of histone modifications and transgene silencing by nickel chloride. Carcinogenesis 27: 1481-1488, 2006.

12. Costa M, Davidson TL, Chen H, et al: Nickel carcinogenesis: epigenetics and hypoxia signaling. Mutat Res 592: 79-88, 2005.

13. Ellen TP, Kluz T, Harder ME, Xiong J and Costa M: Heterochromatinization as a potential mechanism of nickel-induced carcinogenesis. Biochemistry 48: 4626-4632, 2009.

14. Salnikow K and Zhitkovich A: Genetic and epigenetic mechanisms in metal carcinogenesis and cocarcinogenesis: nickel, arsenic, and chromium. Chem Res Toxicol 21: 28-44, 2008.

15. Reddel RR, Ke Y, Gerwin BI, et al: Transformation of human bronchial epithelial cells by infection with SV40 or adenovirus-12 SV40 hybrid virus, or transfection via strontium phosphate coprecipitation with a plasmid containing SV40 early region genes. Cancer Res 48: 1904-1909, 1988.

16. Gao N, Rahmani M, Dent P and Grant S: 2-Methoxyestradiolinduced apoptosis in human leukemia cells proceeds through a reactive oxygen species and Akt-dependent process. Oncogene 24: 3797-3809, 2005.

17. Lin $\mathrm{X}$ and Costa M: Transformation of human osteoblasts to anchorage-independent growth by insoluble nickel particles. Environ Health Perspect 102 (Suppl 3): S289-S292, 1994.
18. Miura T, Patierno SR, Sakuramoto T and Landolph JR: Morphological and neoplastic transformation of $\mathrm{C} 3 \mathrm{H} / 10 \mathrm{~T} 1 / 2 \mathrm{Cl} 8$ mouse embryo cells by insoluble carcinogenic nickel compounds. Environ Mol Mutagen 14: 65-78, 1989.

19. Downward J: Mechanisms and consequences of activation of protein kinase B/Akt. Curr Opin Cell Biol 10: 262-267, 1998.

20. Kau TR, Schroeder F, Ramaswamy S, et al: A chemical genetic screen identifies inhibitors of regulated nuclear export of a Forkhead transcription factor in PTEN-deficient tumor cells. Cancer Cell 4: 463-476, 2003.

21. Manning BD and Cantley LC: AKT/PKB signaling: navigating downstream. Cell 129: 1261-1274, 2007.

22. Stambolic V, Suzuki A, De la Pompa JL, et al: Negative regulation of PKB/Akt-dependent cell survival by the tumor suppressor PTEN. Cell 95: 29-39, 1998.

23. Nave BT, Ouwens M, Withers DJ, Alessi DR and Shepherd PR: Mammalian target of rapamycin is a direct target for protein kinase B: identification of a convergence point for opposing effects of insulin and amino-acid deficiency on protein translation. Biochem J 344 Pt 2: 427-431, 1999.

24. Vogt PK: PI 3-kinase, mTOR, protein synthesis and cancer. Trends Mol Med 7: 482-484, 2001

25. Grimes CA and Jope RS: The multifaceted roles of glycogen synthase kinase 3beta in cellular signaling. Prog Neurobiol 65: 391-426, 2001

26. Datta SR, Dudek H, Tao X, et al: Akt phosphorylation of BAD couples survival signals to the cell-intrinsic death machinery. Cell 91: 231-241, 1997.

27. Yip KW and Reed JC: Bcl-2 family proteins and cancer. Oncogene 27: 6398-6406, 2008.

28. Szatrowski TP and Nathan CF: Production of large amounts of hydrogen peroxide by human tumor cells. Cancer Res 51: 794-798, 1991.

29. Clerkin JS, Naughton R, Quiney C and Cotter TG: Mechanisms of ROS modulated cell survival during carcinogenesis. Cancer Lett 266: 30-36, 2008.

30. Bishop JM: The molecular genetics of cancer. Science 235: 305-311, 1987.

31. Zhang Q, Salnikow K, Kluz T, Chen LC, Su WC and Costa M: Inhibition and reversal of nickel-induced transformation by the histone deacetylase inhibitor trichostatin A. Toxicol Appl Pharmacol 192: 201-211, 2003.

32. Ji WD, Chen JK, Lu JC, Wu ZL, Yi F and Feng SM: Alterations of FHIT gene and P16 gene in nickel transformed human bronchial epithelial cells. Biomed Environ Sci 19: 277-284, 2006.

33. Ji W, Yang L, Yu L, et al: Epigenetic silencing of O6-methylguanine DNA methyltransferase gene in NiS-transformed cells. Carcinogenesis 29: 1267-1275, 2008.

34. Verma R, Ramnath J, Clemens F, Kaspin LC and Landolph JR: Molecular biology of nickel carcinogenesis: identification of differentially expressed genes in morphologically transformed C3H10T1/2 Cl 8 mouse embryo fibroblast cell lines induced by specific insoluble nickel compounds. Mol Cell Biochem 255: 203-216, 2004

35. Landolph JR, Verma A, Ramnath J and Clemens F: Molecular biology of deregulated gene expression in transformed $\mathrm{C} 3 \mathrm{H} /$ 10T1/2 mouse embryo cell lines induced by specific insoluble carcinogenic nickel compounds. Environ Health Perspect 110 (Suppl 5): 845-850, 2002.

36. Chen CH, Lu PJ, Chen YC, et al: FLJ10540-elicited cell transformation is through the activation of PI3-kinase/AKT pathway. Oncogene 26: 4272-4283, 2007.

37. Nomura M, He Z, Koyama I, Ma WY, Miyamoto K and Dong Z: Involvement of the Akt/mTOR pathway on EGF-induced cell transformation. Mol Carcinog 38: 25-32, 2003.

38. Cross DA, Alessi DR, Cohen P,Andjelkovich Mand Hemmings BA: Inhibition of glycogen synthase kinase-3 by insulin mediated by protein kinase B. Nature 378: 785-789, 1995.

39. Harwood AJ: Regulation of GSK-3: a cellular multiprocessor. Cell 105: 821-824, 2001.

40. Polakis P: Wnt signaling and cancer. Genes Dev 14: 1837-1851, 2000.

41. Flacke JP, Kumar S, Kostin S, Reusch HP and Ladilov Y: Acidic preconditioning protects endothelial cells against apoptosis through $\mathrm{p} 38$ - and Akt-dependent Bcl-xL overexpression. Apoptosis 14: $90-96,2009$.

42. Pugazhenthi S, Nesterova A, Sable C, et al: Akt/protein kinase B up-regulates Bcl-2 expression through cAMP-response elementbinding protein. J Biol Chem 275: 10761-10766, 2000. 
43. Qian J, Zou Y, Rahman JS, Lu B and Massion PP: Synergy between phosphatidylinositol 3-kinase/Akt pathway and Bcl-xL in the control of apoptosis in adenocarcinoma cells of the lung. Mol Cancer Ther 8: 101-109, 2009.

44. Bouchard C, Marquardt J, Bras A, Medema RH and Eilers M: Myc-induced proliferation and transformation require Aktmediated phosphorylation of FoxO proteins. EMBO J 23: 2830-2840, 2004
45. Classon $\mathrm{M}$ and Harlow E: The retinoblastoma tumour suppressor in development and cancer. Nat Rev Cancer 2: 910-917, 2002.

46. Iuliano R, Trapasso F, Stella A, et al: Pivotal role of the RB family proteins in in vitro thyroid cell transformation. Exp Cell Res 260: 257-267, 2000.

47. Lin X, Dowjat WK and Costa M: Nickel-induced transformation of human cells causes loss of the phosphorylation of the retinoblastoma protein. Cancer Res 54: 2751-2754, 1994. 